\title{
Location of Resuscitative Endovascular Balloon Occlusion of the Aorta (REBOA) in Zone III Using External Landmark Without Fluoroscopy: A CTA- based Retrospective Study
}

\section{Danlei Weng}

Zhejiang University School of Medicine Second Affiliated Hospital

\section{Anyu Qian}

Zhejiang University School of Medicine Second Affiliated Hospital

Qijing Zhou

Zhejiang University School of Medicine Second Affiliated Hospital

\section{Jiefeng Xu}

Zhejiang University School of Medicine Second Affiliated Hospital

\section{Shanxiang Xu}

Zhejiang University School of Medicine Second Affiliated Hospital

Mao Zhang ( $\sim$ z2jzk@zju.edu.cn )

Zhejiang University School of Medicine Second Affiliated Hospital https://orcid.org/0000-0001-79198213

\section{Original research}

Keywords: Resuscitative balloon occlusion of the aorta, External landmark, Catheter length, Prehospital first aid, Catastrophic hemorrhage

Posted Date: July 28th, 2020

DOI: https://doi.org/10.21203/rs.3.rs-47921/v1

License: (c) (i) This work is licensed under a Creative Commons Attribution 4.0 International License. Read Full License 


\section{Abstract \\ Background}

Resuscitative endovascular balloon occlusion of the aorta (REBOA) can timely prevent the wounded from massive hemorrhage. We aim to study whether the combination of the xiphoid process and the umbilicus could guide the placement of REBOA in zone III without fluoroscopy.

\section{Methods}

We conducted a retrospective study in a university hospital that included 57 subjects who underwent contrast-enhanced computed tomography (CT) from April to December in 2019. External distances and intravascular lengths were measured by three-dimensional reconstruction CT images on the workstation, including the distances from the femoral artery to the xiphoid process (FA-Xi), the midpoint between the xiphoid process and the umbilicus (FA-mXU), the umbilicus (FA-Ui), the midpoint of the zone III (FA-mZIII), the lowest renal artery (FA-LRA), and aortic bifurcation (FA-AB). The relationship between external landmarks and REBOA catheter positioning in zone III was studied, involving the quartering distances between the xiphoid process and the umbilicus, the distance below the xiphoid process and that above the umbilicus. The predicted accuracy and safety margin of the balloon (distal and proximal) were calculated by curvature plane reconstruction. The probability of balloon positioning in zone III using these three methods was compared by Chi square test.

\section{Results}

The average length of aortic zone III was $9.4 \mathrm{~cm}(\mathrm{SD}=10.0)$, and that of FA-mZIII on the right and left sides were $24.4 \mathrm{~cm}(S D=2.1), 23.8 \mathrm{~cm}(S D=2.1)$, respectively. FA-Xi was significantly longer than FALRA, and FA-Ui was significantly shorter than FA-AB $(P<0.05)$. Using the quartering distances between the xiphoid and the umbilicus, the distance below the xiphoid, the distance above the umbilicus to predict the length of REBOA catheter positioning in zone III showed no statistically significant difference. Using FA$\mathrm{mXU}$ to predict the accuracy of catheter positioning in zone III on the left and right sides were $84.2 \%$ and $86 \%$. Although there was a little difference between the left side of FA-mZIII and FA-mXU, there were no statistical differences on the right side.

\section{Conclusions}

The midpoint between the xiphoid process and the umbilicus is a good external landmark to guide the placement of REBOA in zone III without fluoroscopy.

\section{Background}


Massive hemorrhage remains an important cause of death for the injured worldwide. Despite many endeavors, the survival rate remains bleak, and $85 \%$ of hemorrhagic deaths arise within the first six hours $[1,2]$. Rapid and effective hemostasis is a crucial strategy to rescue critically ill patients in prehospital settings. Resuscitative endovascular balloon occlusion of the aorta (REBOA) is a minimally invasive and convenient treatment for non-compressible torso hemorrhage. It can be used by trained physicians to achieve rapid hemostasis, increase arterial pressure, and obtain opportunities for further definitive treatment. However, a series of factors including limited imaging equipment, transportation time and onsite emergency, make it difficult to spread its use in the pre-hospital environment $[3,4]$.

Zone I is described as the region from the left subclavian artery to the celiac trunk, and zone III is described as the region from the lowest renal artery to the aortic bifurcation [5]. It has been suggested that the length of the REBOA catheter positioning in zone I and zone III could be predicted by torso height. Comparing with zone I, zone III has a poor accuracy [6]. The fixed balloon catheter defines zone III as the common segment (236-256 mm) from the superior margin of symphysis pubis, which has been proposed as an alteration to increase the possibility of the placement of REBOA in zone III [7]. Despite the prediction accuracy of zone III could be optimized by this model, Meyer et al. have further verified that the predicted catheter lengths were located partially or completely outside the boundary of the zone III in $50 \%$ of subjects [8]. Thus, in the pre-hospital emergency, a quick and convenient localization method is urgently required to guide the balloon positioning in zone III.

Others have proposed that REBOA can be accomplished by external landmarks without fluoroscopy. Inaba et al. found that the midpoint of the sternum could be used to guide the correct placement of REBOA in zone I via the study of cadavers [9]. Then, Okada et al showed that the safe catheter length positioning in zone I should be between the distance from the femoral artery to suprasternal notch and that to the xiphoid process [10]. In comparison, the current literatures provide less guidance on good practice in regard to the placement of REBOA in zone III by external landmark. Eliason et al placed the balloon to the projection position $5.5 \mathrm{~cm}$ above the umbilicus with marked improvement in predicted accuracy [6]. Yet the best appropriate distance above the umbilicus is still unknown. As the position of xiphoid process is fixed and that of umbilicus is variable due to contour of body, we hypothesize that the combination of the xiphoid process and the umbilicus could guide the placement of REBOA in zone III.

\section{Methods}

This study is a retrospective study, which was certified by the Ethics Committee of the Second Affiliated Hospital, Zhejiang University College of Medicine, and conducted in the radiology department. Data of all patients following no apparent vascular tortuosity by computed tomography (CT) diagnosis aged 1870 years with contrast-enhanced CT images of chest, abdomen, and pelvis from April to December in 2019 were analyzed. Subjects whose images accompanied by vascular malformation, poor contrast enhancement, and history of aortic surgery were precluded. 
Before CT scanning, $70 \mathrm{ml}$ contrast agent iohexol (Omnipaque $370 \mathrm{mg} \mathrm{l} / \mathrm{ml}$, GE Healthcare, Shanghai, China) and $60 \mathrm{ml} 0.9 \%$ saline were injected into the middle elbow vein by a dual-head power injector (Mallinckrodt, American) at a flow rate of $4 \mathrm{ml} / \mathrm{s}$ [11]. A 128-slice dual-energy CT scanner (SOMATOM Definition Flash, Siemens Medical Solutions, Germany) was utilized for the scanning during the arterial phase. The image thickness and interlayer distances are $0.75 \mathrm{~mm}$ and $0.5 \mathrm{~mm}$, respectively. The range of the scan area is from subclavian to bilateral femoral artery. The images were saved to the CT database, then uploaded to a CT workstation (Syngo Via VB10B, Siemens, Germany) following screened by inclusion and exclusion criteria. Effective image reconstruction methods for blood vessels and external landmarks include multiplanar reconstruction, curvature plane reconstruction, and volume rendering.

The external landmarks were measured by volume-rendering reconstruction, referring to the distance from bilateral femoral artery to the xiphoid process (FA-Xi), the midpoint between the xiphoid process and the umbilicus (FA-mXU) and the umbilicus (FA-Ui), the distance from the xiphoid process to the umbilicus. The intravascular regions were measured along the centerline of the blood vessels by multiplanar reconstruction and curvature plane reconstruction, including the lengths from bilateral femoral artery to the left subclavian artery, celiac trunk, lowest renal artery, aortic bifurcation (Fig. 1). Data recording was performed using a standard data record form by a trained clinician and examined for proofreading errors by an experienced imaging expert.

\section{The quartering distances between xiphoid process and umbilicus}

The catheter length in this study was considered as the distance from the femoral artery to the midpoint of the balloon. The balloon length was considered to be $4 \mathrm{~cm}$. The distance between xiphoid process and umbilicus was evenly divided into four parts. The distance of $1 / 4,1 / 2,3 / 4$ was separately added to the distance from the bilateral femoral artery to the umbilicus as the predicted catheter length. The distal safety margin of the balloon was defined as the distance between the superior edge of the balloon and the LRA, and the proximal safety margin of the balloon was defined as the distance between the inferior edge of the balloon and the AB. The distance of the distal safety margin was FA-LRA minus catheter length minus $2 \mathrm{~cm}$, the distance of the proximal safety margin of the balloon was catheter length minus FA-AB minus $2 \mathrm{~cm}$ (Fig. 2). (Bilateral balloon safety margin must be greater than or equal to 0 ). Simultaneously, the prediction accuracy was calculated.

\section{The distance below the xiphoid}

The distance from the bilateral femoral artery to the xiphoid was measured on the workstation, then separately minus $1,2,3,4,5-12 \mathrm{~cm}$ as the predicted catheter length. Distal and proximal balloon safety margin distances, as well as prediction accuracy, were calculated by using this catheter length.

\section{The distance above the umbilicus}


The distance from the bilateral femoral artery to the umbilicus was measured on the workstation, then added 1, 2, 3, 4, 5-12cm in sequence as the predicted catheter length. Similarly, distal and proximal balloon safety margin distances, as well as prediction accuracy, were calculated with this catheter length.

\section{Statistical Analysis}

The measured distances and lengths were described as mean (standard deviation SD). Age, sex, height, and BMI were used to describe the individual characteristics. Data were inserted into an Excel database (Excel 2019, Microsoft), and statistical analysis was performed by using SPSS statistics 20.0 (SPSS Inc., Chicago, IL, USA). Tests comparing bilateral data differences were conducted by using paired-sample ttests if Shapiro-Wilk (S-W) test indicates that the data were normally distributed. The difference between the three predicted methods was compared by the Chi-Square test. Differences were declared to be significant when $p$-values were less than $0.05(P<0.05)$.

\section{Result}

The contrast-enhanced CT scans of the chest, abdominal, and pelvic were available for analysis on 77 subjects from April to December in 2019. We identified 70 subjects who fulfilled the inclusion criteria. Of these, 7 subjects were diagnosed with aortic dissection or aortic aneurysm and 6 subjects lost general information. Ultimately, 57 subjects were enrolled in the study (Fig. 3).

The individual characteristics of the subjects were described in Table 1. The mean age of all patients was 60 years $(S D=9.4)$, mean height was $164 \mathrm{~cm}(\mathrm{SD}=8.6)$, and mean BMI was $23.2 \mathrm{~kg} / \mathrm{m}^{2}(\mathrm{SD}=3.0), 70 \%$ of the subjects were male $(n=40)$. The length of zone I was $24.7 \mathrm{~cm}(S D=2.2)$ which is the longest region in the aorta, while zone III had only a length of $9.4 \mathrm{~cm}(S D=1.0)$. The FA-AB on the right side was $5 \mathrm{~mm}$ longer than that of the left side. Similarly, the FA-LRA on the right side was $5 \mathrm{~mm}$ longer than that of the left side. In general, the right length of REBOA catheter positioning in zone III was slightly longer compared with the left side. The average length from the femoral artery to the midpoint of zone III on the right and left sides were $24.4 \mathrm{~cm}(S D=2.1), 23.8 \mathrm{~cm}(S D=2.1)$, respectively (Table 2). The box plots can be used to describe the distribution of lengths ranging from the bilateral femoral artery to the key anatomical structure (Fig. 4a). 
Table 1

Clinical Characteristics of the 57 subjects

\begin{tabular}{|lllll|}
\hline Characteristic & Mean (SD) & Minimum & Median & Maximum \\
\hline Men $(\mathrm{n}=40)$ & & & & \\
\hline Age, $y$ & $59.2(10.6)$ & 28 & 63 & 70 \\
\hline Height, $\mathrm{cm}$ & $167.0(8.4)$ & 130.0 & 170.0 & 183.0 \\
\hline BMl, $\mathrm{kg} / \mathrm{m}^{2}$ & $23.7(2.5)$ & 17.3 & 23.7 & 29.7 \\
\hline Women $(\mathrm{n}=17)$ & & & & \\
\hline Age, $\mathrm{y}$ & $61.8(5.3)$ & 49 & 63 & 69 \\
\hline Height, $\mathrm{cm}$ & $158.0(5.0)$ & 150.0 & 160.0 & 165.0 \\
\hline BMl, $\mathrm{kg} / \mathrm{m}^{2}$ & $22.0(3.6)$ & 16.4 & 21.8 & 29.4 \\
\hline Total $(\mathrm{n}=57)$ & & & & \\
\hline Age, $\mathrm{y}$ & $60.0(9.4)$ & 28 & 63 & 70 \\
\hline Height, cm & $164.3(8.6)$ & 130.0 & 165.0 & 183.0 \\
\hline BMl, $\mathrm{kg} / \mathrm{m}^{2}$ & $23.2(3.0)$ & 16.4 & 23.1 & 29.7 \\
\hline BMl, body mass index. & & & \\
\hline
\end{tabular}


Table 2. Measurement of intravascular landmarks

Intravascular measurement

Mean (SD) Minimum Median Maximum $P$ value ${ }^{*}$

Vessel Lengths(cm)

Left FA to LSCA

$56.6(3.9)$

50.3

55.7

68.2

0.019

Right FA to LSCA

$57.1(4.0)$

50.1

56.4

69.5

0.028

Left FA to Mid-Zone I

44.3(3.1)

38.3

43.4

53.0

0.035

Right FA to Mid-Zone I

44.8(3.1)

38.9

44.0

54.4

0.080

Left FA to CT

31.9(2.4)

26.6

31.5

38.2

0.246

Right FA to CT

$32.4(2.4)$

27.7

32.1

39.6

0.490

Left FA to LRA

28.5(2.2)

22.1

28.2

33.1

0.601

Right FA to LRA

29.0(2.2)

$23 . .3$

28.9

34.2

0.809

Left FA to Mid-Zone III

23.8(2.1)

18.0

23.3

28.5

0.405

Right FA to Mid-Zone III

24.4(2.1)

19.4

24.1

28.9

0.658

Left $F A$ to $A B$

19.2(2.0)

13.9

18.7

23.8

0.310

Right $F A$ to $A B$

19.7(2.1)

15.4

19.7

24.1

0.401

Zone Lengths $(\mathrm{cm})$

\begin{tabular}{llllll} 
Zone I & $24.7(2.2)$ & 20.8 & 24.8 & 29.7 & 0.458 \\
\hline Zone II & $3.4(0.8)$ & 1.9 & 3.3 & 5.7 & 0.102 \\
\hline Zone III & $9.4(1.0)$ & 7.4 & 9.3 & 12.2 & 0.627
\end{tabular}

FA, Femoral artery; AB, Aortic bifurcation; LRA, Lowest renal artery; CT, Celiac trunk; LSCA, Left subclavian artery; Zone I, defined as the distance from left subclavian artery to celiac trunk; Zone III, defined as the distance from lowest renal artery to aortic bifurcation. $P$ value $\llbracket$ date were considered to satisfy the normal distribution if $P>0.05$ ( $S$-W test). 
Table 3.Measurement of key anatomical landmarks from the femoral artery

\begin{tabular}{llllll} 
Distances(cm) & Mean (SD) & Minimum & Median & Maximum & $P$ value \\
\hline Right & & & & & \\
\hline FA to LSCA & $57.2(4.0)$ & 50.1 & 56.4 & 69.5 & 0.028 \\
\hline FA to SSN & $50.8(3.1)$ & 39.8 & 51.4 & 56.5 & 0.014 \\
\hline FA to MS & $42.1(2.8)$ & 31.8 & 42.8 & 46.8 & 0.003 \\
\hline FA to CT & $32.4(2.4)$ & 27.7 & 32.1 & 39.6 & 0.490 \\
\hline FA to Xi & $33.0(2.8)$ & 23.2 & 33.4 & 37.7 & 0.009 \\
\hline FA to LRA & $29.0(2.2)$ & 23.3 & 28.9 & 34.2 & 0.809 \\
\hline FA to MXU & $24.1(2.0)$ & 17.4 & 24.4 & 27.7 & 0.037 \\
\hline FA to Ui & $15.5(1.5)$ & 11.6 & 15.7 & 18.2 & 0.289 \\
\hline FA to AB & $19.7(2.1)$ & 15.4 & 19.7 & 24.1 & 0.401
\end{tabular}

Left

\begin{tabular}{|llllll|}
\hline FA to LSCA & $56.6(3.9)$ & 50.3 & 55.7 & 68.2 & 0.019 \\
\hline FA to SSN & $51.0(3.2)$ & 39.7 & 51.5 & 56.5 & 0.015 \\
\hline FA to MS & $42.3(2.8)$ & 31.3 & 43.0 & 46.7 & 0.001 \\
\hline FA to CT & $31.9(2.4)$ & 26.6 & 31.5 & 38.2 & 0.246 \\
\hline FA to Xi & $33.2(2.7)$ & 23.0 & 33.2 & 37.9 & 0.008 \\
\hline FA to LRA & $28.5(2.2)$ & 22.1 & 28.2 & 33.1 & 0.601 \\
\hline FA to MXU & $24.4(1.9)$ & 17.8 & 24.6 & 27.8 & 0.043 \\
\hline FA to Ui & $15.9(1.5)$ & 12.2 & 16.0 & 19.1 & 0.733 \\
\hline FA to AB & $19.2(2.0)$ & 13.9 & 18.7 & 23.8 & 0.310 \\
\hline
\end{tabular}

FA, Femoral artery; LSCA, Left subclavian artery; SSN, Suprasternal notch; MS, Mid-sternum; CT, Celiac trunk; Xi, Xiphoid process; LRA, Lowest renal artery; MXU, Midpoint of the distance from xiphoidprocess to umbilicus; Ui, Umbilicus; $\mathrm{AB}$, Aortic bifurcation. $P$ value ${ }^{\star} \llbracket$ date were considered to satisfy the normal distribution if $P>0.05$ ( $S$ - $W$ test). 


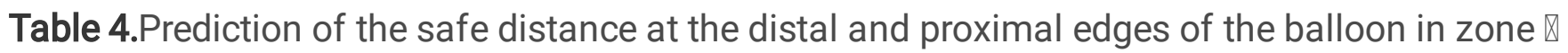

\begin{tabular}{|c|c|c|c|}
\hline & $\begin{array}{l}\text { The midpoint of } \\
\text { xiphoid and } \\
\text { umbilicus }^{*}\end{array}$ & $\begin{array}{l}\text { the distance } \\
\text { above the } \\
\text { umbilicus }^{*}\end{array}$ & $\begin{array}{l}\text { The distance } \\
\text { below the } \\
\text { xiphoid }^{\star}\end{array}$ \\
\hline \multicolumn{4}{|l|}{$\begin{array}{l}\text { Distal safe } \\
\text { distance }(\mathrm{cm})\end{array}$} \\
\hline Right femoral artery & $3.0 \pm 1.7$ & $2.7 \pm 1.8$ & $3.0 \pm 1.9$ \\
\hline Left femoral artery & $2.5 \pm 1.7$ & $3.4 \pm 1.9$ & $2.7 \pm 1.8$ \\
\hline \multicolumn{4}{|l|}{ Proximal safe distance $(\mathrm{cm})$} \\
\hline Right femoral artery & $2.4 \pm 1.7$ & $2.8 \pm 1.6$ & $2.4 \pm 1.9$ \\
\hline Left femoral artery & $3.1 \pm 1.6$ & $2.1 \pm 1.5$ & $2.8 \pm 1.9$ \\
\hline \multicolumn{4}{|l|}{ Prediction accuracy (\%) } \\
\hline Right femoral artery $(n=57)$ & $49(86.0)$ & $51(89.5)$ & $47(82.5)$ \\
\hline Left femoral artery $(n=57)$ & $48(84.2)$ & $50(87.7)$ & $46(80.7)$ \\
\hline \multicolumn{4}{|c|}{$\begin{array}{l}\text { Safe distance was described as mean } \pm \text { standard deviation. The measured catheter length was } \\
\text { defined as the distance from the femoral artery to the midpoint of the balloon. Distal safe distance } \\
\text { was the distance from the distal edge of the balloon to the lowest renal artery. Proximal safe distance } \\
\text { was the distance from the proximal edge of the balloon to the aorticbifurcation. The right distance } \\
\text { above the umbilicus is } 9 \mathrm{~cm} \text { and the left distance above the umbilicus is } 7 \mathrm{~cm} \text {. The bilateral distance } \\
\text { below the xiphoid is } 9.5 \mathrm{~cm} \text {. }{ }^{*} \text { Chi-Square test, } P>0.05 \text {, no statistically significant. }\end{array}$} \\
\hline
\end{tabular}

The distance from the bilateral femoral artery to the external landmarks were measured (Table 3). The right and left distance of the FA-Xi were $33.0 \mathrm{~cm}(\mathrm{SD}=2.8)$ and $33.2 \mathrm{~cm}(\mathrm{SD}=2.7)$, of the FA-Ui were $15.5 \mathrm{~cm}(S D=1.5)$ and $15.9 \mathrm{~cm}(S D=1.5)$. The right and left length of the FA-LRA were $29.0 \mathrm{~cm}(\mathrm{SD}=$ 2.2) and $28.5 \mathrm{~cm}(S D=2.2)$, of the FA-AB were $19.7 \mathrm{~cm}(S D=2.1)$ and $19.2 \mathrm{~cm}(S D=2.0)$. It was evident that the FA-Xi was longer than the FA-LRA and the FA-Ui was shorter than the FA-AB (paired two-tailed test, $P<0.05)$ (Fig. 4a). Although there was a little difference between the left side of FA-mZIII and FA-mXU (paired two-tailed test, $P=0.04$ ) (Fig. 4b), there were no statistical differences on the right side (paired two-tailed test, $P>0.05$ ) (Fig. 4b).

The 2 equal distances of xiphoid and umbilicus (the midpoint between the xiphoid process and the umbilicus) were added to the bilateral distances from the femoral artery to the umbilicus with acceptable predicted accuracy (right: $86.0 \%$, left:84.2\%). The average of distal balloon safety margin distances with standard deviation on the right and left sides were $3.0 \mathrm{~cm}(S D=1.7)$ and $2.5 \mathrm{~cm}(\mathrm{SD}=1.7)$, respectively. 
The proximal balloon safety margin distances on the right and left sides were $2.4 \mathrm{~cm}(\mathrm{SD}=1.7)$ and $3.1 \mathrm{~cm}(S D=1.6)$. The distance from the femoral artery to $9 \mathrm{~cm}$ above the umbilicus on the right side was used to predict the length of REBOA catheter positioning in zone III with the highest accuracy (89.5\%). The distal and proximal balloon safety margin distance on the right side were $2.7 \mathrm{~cm}(S D=1.8)$ and $2.8 \mathrm{~cm}(S D=1.6)$, respectively. In contrast, the distance from the femoral artery to $7 \mathrm{~cm}$ above the umbilicus on the left side reached an accuracy of $87.7 \%$. The distal and proximal balloon safety margin distance on the left side were $3.4 \mathrm{~cm}(S D=1.9)$ and $2.1 \mathrm{~cm}(S D=1.5)$, respectively. The predicted accuracy of the distance from the femoral artery to $9.5 \mathrm{~cm}$ below the xiphoid on right and left sides were $82.5 \%$ and $80.7 \%$, respectively. The distal balloon safety margin distances on the right and left sides were $3.0 \mathrm{~cm}(\mathrm{SD}=1.9)$ and $2.7 \mathrm{~cm}(\mathrm{SD}=1.8)$, respectively. The proximal balloon safety margin distances on the right and left sides were $2.4 \mathrm{~cm}(\mathrm{SD}=1.9)$ and $2.8 \mathrm{~cm}(\mathrm{SD}=1.9)$, respectively. The bilateral predicted accuracy of catheter length positioning in zone III were no significant difference in statistical analysis using the above three predicted methods (Chi-Square test, $p>0.05$ ) (Table. 4) (Fig. 5).

\section{Discussion}

In our study, the xiphoid process and the umbilicus has been demonstrated to be involved in REBOA positioning in zone III with acceptable predicted accuracy (right: $86.0 \%$, left:84.2\%). FA-Xi was longer than FA-LRA and FA-Ui was shorter than the FA-AB. Hence, the length of REBOA catheter positioning in zone III should range from FA-Xi to FA-Ui. The combination of xiphoid process and umbilicus can be used to guide the placement of REBOA in zone III without fluoroscopy.

At present, studies have shown that severe pelvic fractures are regularly accompanied by arterial injury, and the mortality rate can achieve $32 \%$ when hemorrhagic shock occurs [12]. Timely and effective treatment of vascular hemorrhage is critical to prevent death from hemorrhagic shock. Clinically, it is possible to intervene early through pelvic packing, and then be treated with interventional embolization to further control catastrophic hemorrhage [13]. However, it is almost unrealistic to achieve complete hemostasis by means of vascular embolization in the pre-hospital environment. REBOA can achieve rapid hemostasis in the pre-hospital settings to maintain central arterial pressure and stabilize vital signs. But due to the limited transport times and pre-hospital environment, the placement of REBOA in zone III is still not ideal at present.

Although it has been proposed the estimation of the catheter length positioning in zone III by torso height, the result was just passable [6]. Wessels et al tried to apply radiofrequency identification to predict the location of balloon in zone III on the cadaver with an accuracy of only $16 \%$ [14]. It is also recommended to assemble various individual information (gender, smoking, diabetes mellitus, etc.) to estimate catheter length through multi-factor correlation analysis [15]. Considering the integrity of information and the accuracy of calculation, it may hamper its wide application in the pre-hospital environment. Recently, New technologies such as Gamma probe and the magnetically trackable device have been applied into the balloon placement of the REBOA without fluoroscopy, yet these studies are still in the animal experimental stage $[16,17]$. Thus, it is unclear whether these findings are applicable to human being. The 
positioning of the balloon guided by anatomical structures has attracted much attention. Measuring the distance between external landmarks can directly determine the inserted catheter length for the sake of balloon positioning, which is a practical approach.

Some researchers have struggled to utilize anatomical structures or other strategies to guide the right placement of REBOA. Martinelli et al. proposed that the catheter was inserted $50 \mathrm{~cm}$ toward the cephalic, filled with the saline, withdrawn until it was wedged in the aortic bifurcation, and then moved $5 \mathrm{~cm}$ to the cephalic [18]. However, it should be noted that the withdrawal process may provoke vascular endothelial damage and the relevant imaging equipment is required for real-time positioning. Recently, It has been proposed to estimate the balloon length by torso height. The accuracy of balloon placement in the target region is appreciable-the accuracy of zone I and zone III are $100 \%, 74 \%$, respectively [6]. We have to admit, however, that the accuracy of the catheter length positioning in zone III is not very satisfactory. In our study, the catheter length positioning in zone III can be quickly estimated according to the external landmarks. Of these, we found that the aortic length was shorter in those patients with short stature. If the fixed catheters continued to be applied in zone III at this time, it may cause long-term ischemic necrosis of vital organs. Inaba and Okada proposed that sternum was likely to be used to guide the catheter length positioning in zone I $[9,10]$, yet there is no further study on the relevant anatomical structure of zone III.

Through analysis of the CTA measurement data, our study further verified that the accuracy of the catheter length positioning in zone I was up to be $100 \%$ by the midpoint of the sternum. Besides, we utilized three measurement methods to compare the difference of predicted accuracy in zone III and found that the midpoint of the xiphoid process and the umbilicus, $7 \mathrm{~cm}$ or $9 \mathrm{~cm}$ above the umbilicus, and $9.5 \mathrm{~cm}$ below the xiphoid process were proven no significant difference. Considering the characteristics of simple operation and quick location in the pre-hospital environment, the midpoint of the xiphoid process and the umbilicus is the preferred method to predict the appropriate catheter length. The optimal distances above umbilicus on the left and right sides are not the same, which may be explained by the difference in bilateral anatomical structures. Interestingly, the optimal distance below the xiphoid process and above the umbilicus is close to half of the distance between the xiphoid process and the umbilicus $(17.9 \mathrm{~cm}(\mathrm{SD}=2.1))$. Thus, we have reason to believe that the midpoint of the umbilicus and xiphoid process may be a useful external landmark for the rapid judgment of catheter length positioning in zone III.

Rapid determination of the catheter length positioning in zone III is significantly imperative for the prehospital patients who suffered from catastrophic hemorrhage due to the lower limb and pelvis. Although the position of the balloon can be confirmed by X-ray and other imaging examinations after REBOA balloon being filled, it is impractical to achieve this strategy in the pre-hospital environment. For patients with non-compressible torso hemorrhage, the long-time malposition of balloon not only fail to achieve effective hemostasis in the target region, but also largely cause balloon-related complications. As the White Book states, the maximum transportation time for a major trauma patient from the scene should not exceed 30 minutes [19]. We found that the right FA-mZIII and FA-mXU were shown no significant

Page $11 / 18$ 
difference in statistical analysis, and FA-mXU could be used to guide the inserted catheter length positioning in zone III. It is well known that the length of zone III is about $8-9 \mathrm{~cm}$ without a large faulttolerance range as zone I. "Too long" and "too short" are likely to lead to disastrous consequences. "Too long" means that the inferior edge of the catheter balloon is easy to cross the lowest renal artery and block the blood supply of vital organs, which immensely reduces the blocking time of REBOA and drives visceral ischemic necrosis and reperfusion injury in severe cases. "Too short" means that the superior edge of the catheter balloon fails to exceed the aortic bifurcation, which can easily lead to vascular dissection or even amputation, thus fail to take full advantage of REBOA in the rapid control of catastrophic hemorrhage. As a result, the appropriate catheter length positioning in zone III is essential to rapid hemostasis in a critical situation.

Finally, several limitations to this study should be considered. First, these conclusions were based on the responses of CT three-dimensional reconstruction and might not vividly reflect measurement processes on the intact body. The positioning of body surface markers (such as umbilicus) may be affected by body shape and body position.

For special groups, such as obesity and pregnant patients, some extra imaging methods must be equipped to avoid possible injuries and confirm precise positioning before inflation. Second, the catheter length estimated by the external landmark may be different from the actual length running in the vascular. The actual catheter length may also be affected by the blood flow velocity and the degree of vascular curvature. Third, our subjects were Chinese population, so it is not known whether our results apply to other groups. Larger studies with longer follow-up are needed to study whether exists other factors affecting the prediction of catheter length in zone III.

\section{Conclusion}

The midpoint of the xiphoid process and the umbilicus is a good external landmark for predicting the balloon position in zone III. We are convinced that it could be a relatively reliable and convenient way to guide the right placement of REBOA in zone III without fluoroscopy in the pre-hospital environment.

\section{Abbreviations}

\section{REBOA}

Resuscitative endovascular balloon occlusion of the aorta; CT:Computed tomography; FA:Femoral artery; Xi:Xiphoid process; mXU:Midpoint between the xiphoid process and the umbilicus; Ui:Umbilicus; mZIII:Midpoint of the zone III; BMI:Body mass index; LSCA:Left subclavian artery; CT:Celiac trunk; LRA:Lowest renal artery; AB:Aortic bifurcation; LFA:Left femoral artery; RFA:Right femoral artery.

\section{Declarations}




\section{Ethics approval and consent to participate}

This study was approved by the Ethics Committee of the Second Affiliated Hospital of Zhejiang University School of Medicine (approval number: 2019380).

\section{Consent for publication}

Not applicable.

\section{Availability of data and materials}

All the data are available and will be submitted if required.

\section{Competing interests}

The authors declare that they have no competing interests.

\section{Funding}

Mao Zhang is funded by the Key Program Co-sponsored by Zhejiang Province and National Health and Family Planning Commission of China (2018271879) and the Welfare Scientific Research Project of Zhejiang Province (LGF18H150003). Anyu Qian is funded by the Welfare Scientific Research Project of Zhejiang Province (LGD19H150003). These agencies provided financial support but were not involved in any other aspect of the research.

\section{Authors' contributions}

The corresponding author (Zhang Mao and Xu Shan-xiang) was in charge of study design and data analysis. The first author (Weng Dan-lei) was responsible for data collection, manuscript writing and cooperated with the rest three authors (Qian An-yu, Zhou Qi-jing and Xu Jie-feng) in data analysis and correction work. All authors participated in the critical revision of the manuscript, and all authors approved the final version to be submitted.

\section{Acknowledgements}

Not applicable.

\section{Authors' information}

${ }^{1}$ Department of Emergency Medicine, Second Affiliated Hospital, Zhejiang University School of Medicine, Hangzhou 310009, China. ${ }^{2}$ Institute of Emergency Medicine, Zhejiang University, Hangzhou 310009, 
China. ${ }^{3}$ Department of Radiology, Second Affiliated Hospital, Zhejiang University School of Medicine, Hangzhou 310009, China.

\section{References}

1. Cannon JW, Khan MA, Raja AS, Cohen MJ, Como JJ, Cotton BA, et al. Damage control resuscitation in patients with severe traumatic hemorrhage: a practice management guideline from the eastern association for the surgery of trauma. J Trauma Acute Care Surg. 2017;82(3):605-17.

2. Fox EE, Holcomb JB, Wade CE, Bulger EM, Tilley BC. Earlier endpoints are required for hemorrhagic shock trials among severely injured patients. Shock. 2017;47(5):567-73.

3. Brede JR, Lafrenz T, Krüger AJ, Søvik E, Steffensen T, Kriesi C, et al. Resuscitative endovascular balloon occlusion of the aorta (REBOA) in non-traumatic out-of-hospital cardiac arrest: evaluation of an educational programme. BMJ Open. 2019;9(5):e027980.

4. Henry R, Matsushima K, Henry RN, Wong V, Warriner Z, Strumwasser A, et al. Who would have benefited from the prehospital use of resuscitative endovascular balloon occlusion of the aorta (REBOA)? an autopsy study. J Am Coll Surg. 2019;229(4):383-8.e1.

5. Stannard A, Eliason JL, Rasmussen TE. Resuscitative endovascular balloon occlusion of the aorta (REBOA) as an adjunct for hemorrhagic shock. J Trauma. 2011;71(6):1869-72.

6. Eliason JL, Derstine BA, Horbal SR, Wang NC, Holcombe SA, Chiu CH, et al. Computed tomography correlation of skeletal landmarks and vascular anatomy in civilian adult trauma patients: implications for resuscitative endovascular balloon occlusion of the aorta. J Trauma Acute Care Surg. 2019;87(1S Suppl 1):138-45.

7. Pezy P, Flaris AN, Prat NJ, Cotton F, Lundberg PW, Caillot J-L, et al. Fixed-distance model for balloon placement during fluoroscopy-free resuscitative endovascular balloon occlusion of the aorta in a civilian population. JAMA Surg. 2017;152(4):351-8.

8. Meyer DE, Mont MT, Harvin JA, Kao LS, Wade CE, Moore LJ. Catheter distances and balloon inflation volumes for the ER-REBOA ${ }^{\mathrm{TM}}$ catheter: a prospective analysis. Am J Surg. 2020;219(1):140-4.

9. Linnebur M, Inaba K, Haltmeier T, Rasmussen TE, Smith J, Mendelsberg R, et al. Emergent non-imageguided resuscitative endovascular balloon occlusion of the aorta (REBOA) catheter placement: a cadaver-based study. J Trauma Acute Care Surg. 2016;81(3):453-7.

10. Okada Y, Narumiya H, Ishi W, liduka R. Anatomical landmarks for safely implementing resuscitative balloon occlusion of the aorta (REBOA) in zone 1 without fluoroscopy. Scand J Trauma Resusc Emerg Med. 2017;25(1):63.

11. Liu X, He Y, Zhu Q, Gao F, He W, Yu L, et al. Supra-annular structure assessment for self-expanding transcatheter heart valve size selection in patients with bicuspid aortic valve. Catheter Cardiovasc Interv. 2018;91(5):986-94.

12. Costantini TW, Coimbra R, Holcomb JB, Podbielski JM, Catalano R, Blackburn A, et al. Current management of hemorrhage from severe pelvic fractures: results of an American association for the 
surgery of trauma multi-institutional trial. J Trauma Acute Care Surg. 2016;80(5):717-23. discussion 723-5.

13. Burlew CC, Moore EE, Smith WR, Johnson JL, Biffl WL, Barnett CC, Stahel PF. Preperitoneal pelvic packing/external fixation with secondary angioembolization: optimal care for life-threatening hemorrhage from unstable pelvic fractures. J Am Coll Surg. 2011;212(4):628-35. discussion 635-7.

14. Wessels LE, Wallace JD, Bowie J, Butler WJ, Spalding C, Krzyzaniak M. Radiofrequency identification of the ER-REBOA: confirmation of placement without fluoroscopy. Mil Med. 2019;184(3-4):e285-9.

15. MacTaggart JN, Poulson WE, Akhter M, Seas A, Thorson K, Phillips NY, et al. Morphometric roadmaps to improve accurate device delivery for fluoroscopy-free resuscitative endovascular balloon occlusion of the aorta. J Trauma Acute Care Surg. 2016;80(6):941-6.

16. Park Y, Jo YG, Choi K-H, Kim M, Kim J. Gamma probe-guided confirmation of balloon placement in endovascular procedures. J Trauma Acute Care Surg. 2019;86(6):994-1000.

17. Rezende-Neto JB, Ravi A, Semple M. Magnetically trackable resuscitative endovascular balloon occlusion of the aorta: a new non-image-guided technique for resuscitative endovascular balloon occlusion of the aorta. J Trauma Acute Care Surg. 2020;88(2):e87-91.

18. Martinelli T, Thony F, Decléty P, Sengel C, Broux C, Tonetti J, et al. Intra-aortic balloon occlusion to salvage patients with life-threatening hemorrhagic shocks from pelvic fractures. J Trauma. 2010;68(4):942-8.

19. Koller M, Ernstberger A, Zeman F, et al. Outcome after polytrauma in a certified trauma network: comparing standard vs. maximum care facilities concept of the study and study protocol (POLYQUALY). BMC Health Serv Res, 2016;16(242).

\section{Figures}
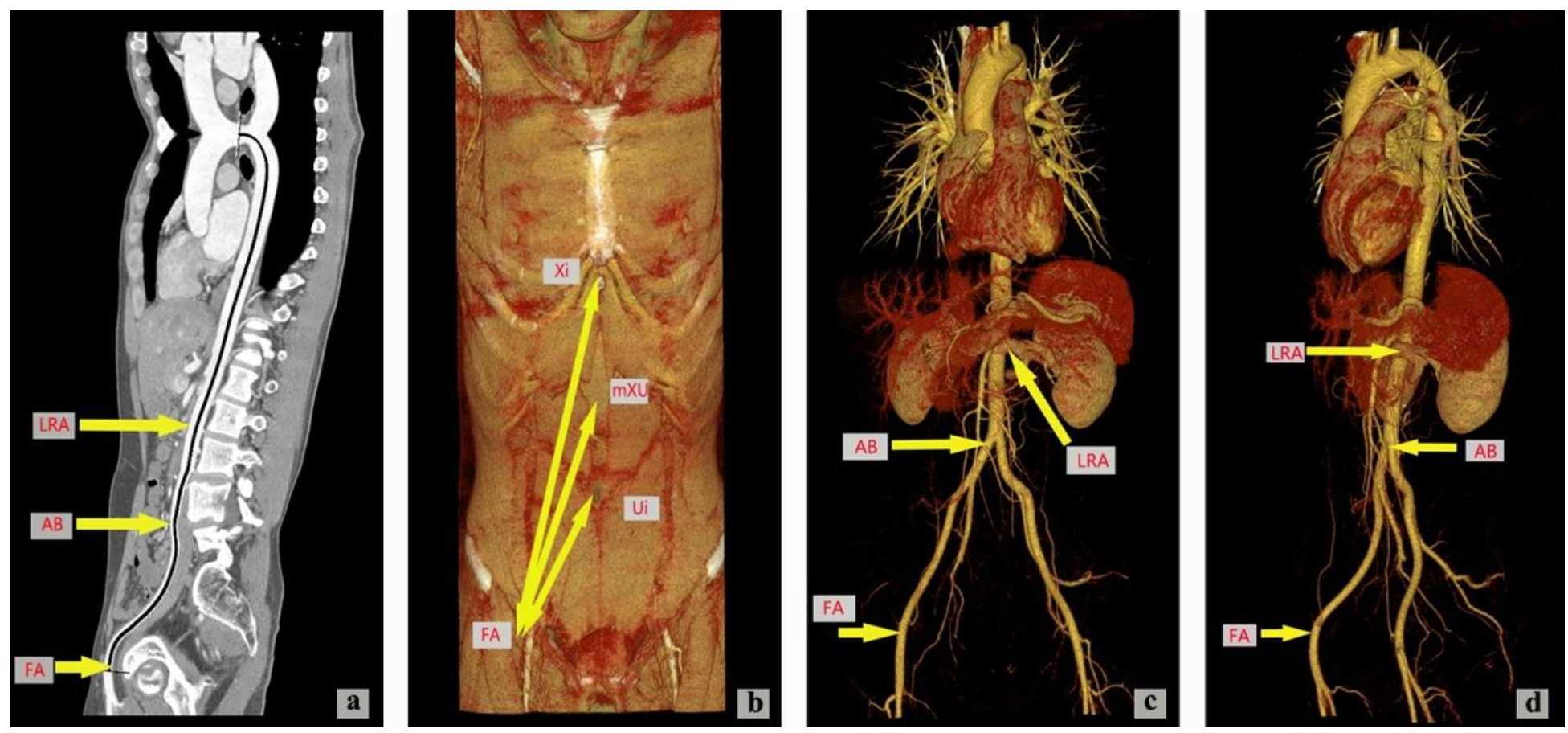
Figure 1

Multiplanar reconstruction, curvature plane reconstruction and volume-renderingimages.(a)Multiplanar reconstruction and curvature plane reconstruction were used to measure the length from the bilateral femoral artery (FA) to the lowest renal artery (LRA) and the aortic bifurcation (AB). (b)Volume-rendering was used to measure the distance from the bilateral femoral artery $(F A)$ to the xiphoid process $(\mathrm{Xi})$, the midpoint of the distance from xiphoid process to umbilicus (mXU) and the umbilicus (Ui).(cखd). Threedimensional vascular imaging technology was used to depict the front and side images of the total aorta.



a

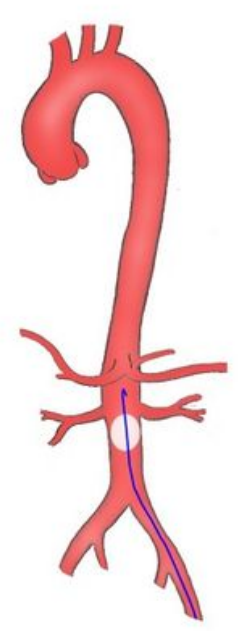

b

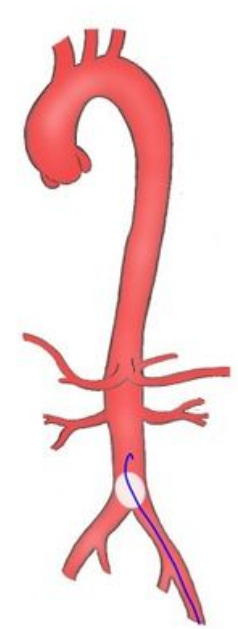

C

\section{Figure 2}

The schematic diagram of aortic balloon occlusion. (a)Division of aorta and location of related blood vessels; (b) The superior edge of the aortic balloon coincides with the lowest renal artery;(c) The inferior edge of the aortic balloon coincides with the aortic bifurcation. 


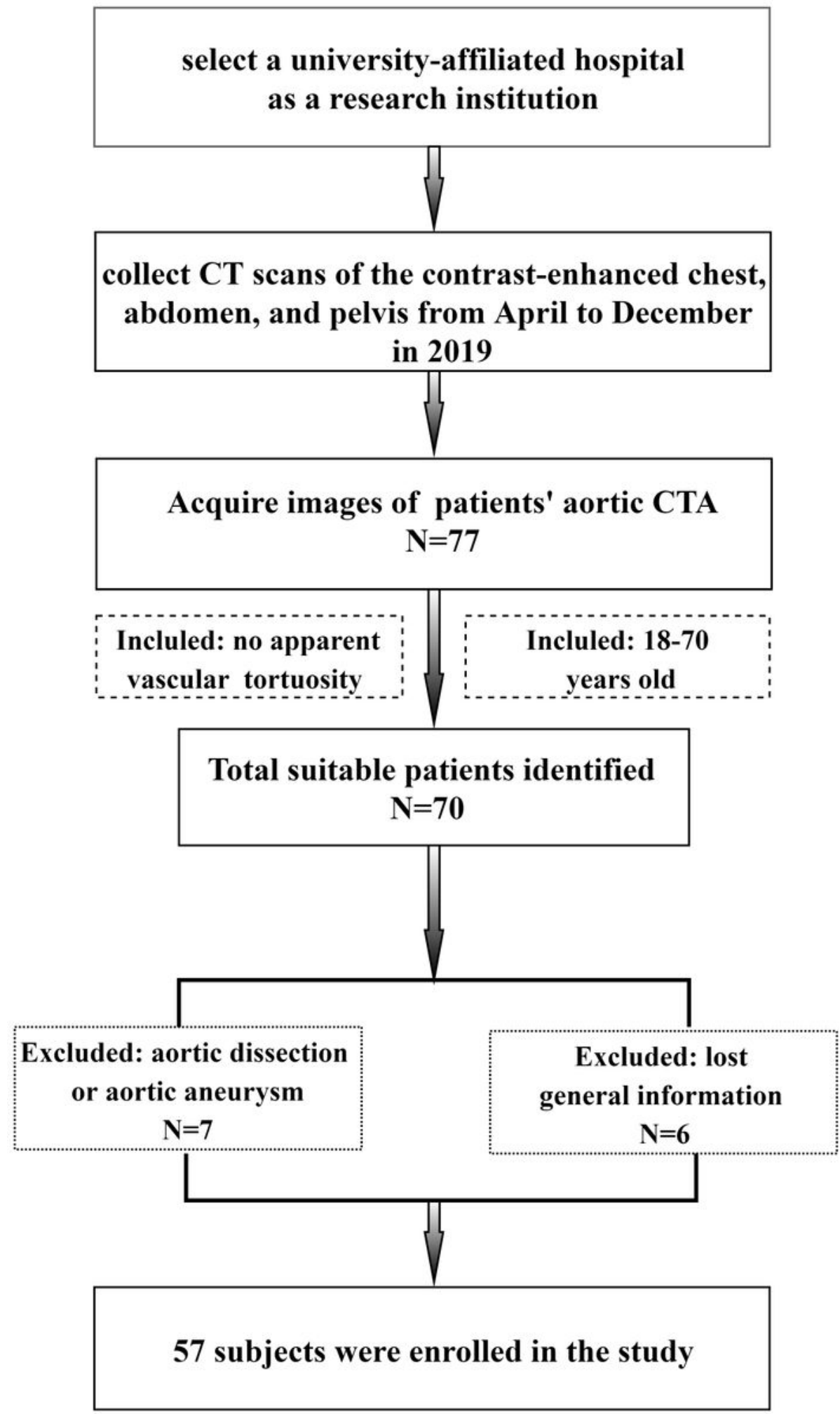

Figure 3

Flow chart for screening subjects. 

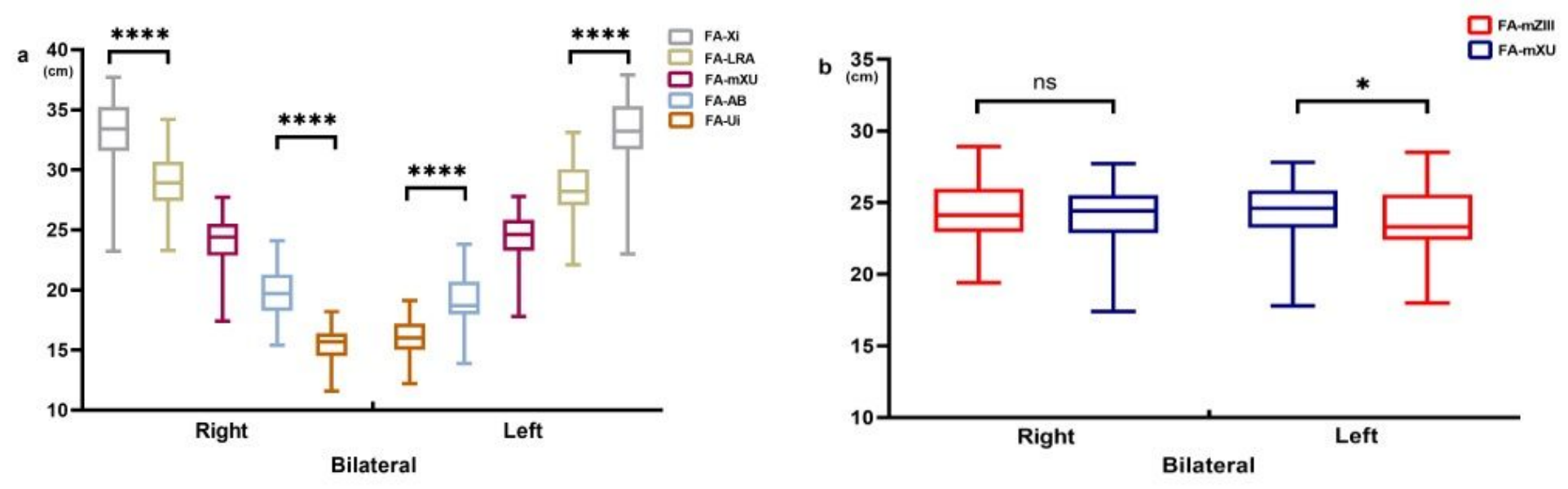

Figure 4

Description of measured values distribution in this study. (a)The box plot describes the fact that the FA-Xi was remarkably longer than the FA-LRA and the FA-Ui was remarkably shorter than the FA-AB (paired twotailed test, $p<0.001$ ).FA-mXU is between FA-LRA and FA-AB. (b)The box plot shows that FA-mZIII and FA$m X U$ on the left side differ slightly (paired two-tailed test, $p=0.04$ ), and the right side is shown no significant difference (paired two-tailed test, $p>0.05$ ). FA-LRA means the length from the femoral artery to the lowest renal artery. FA-MXU means the distance from the femoral artery to the midpoint of the xiphoid process and umbilicus. FA-AB means the length from the femoral artery to aortic bifurcation. FA-Ui means the distance from the femoral artery to the umbilicus. FA-mZIII means the length from the femoral artery to the midpoint of the of zone III. FA, Femoral artery; Xi, Xiphoid process; LRA, Lowest renal artery; mXU, Midpoint of the xiphoid process and umbilicus; AB, Aortic bifurcation; Ui, Umbilicus; mZIII, Midpoint of the of zone III;****: $p<0.0001 ; *$ : $p<0.05$;NS, non-significant difference.
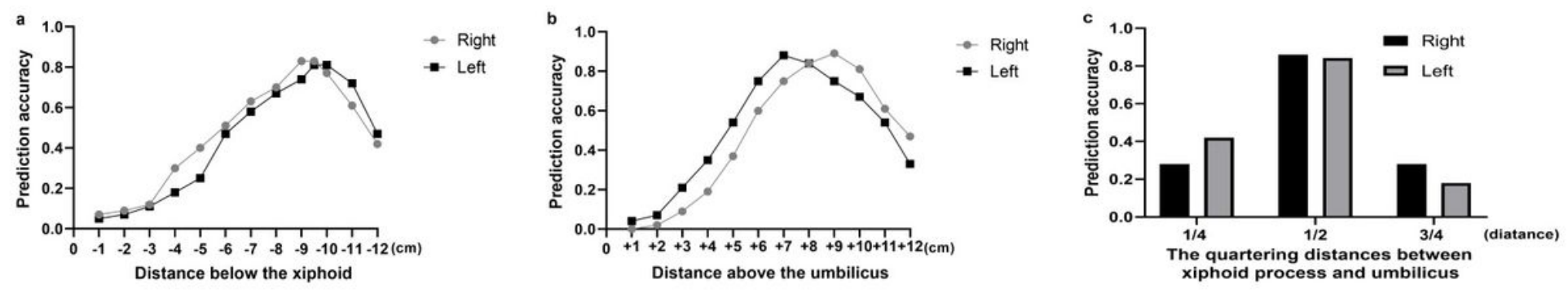

Figure 5

Accuracy of different body surface distances and the predicted catheter length positioning in zone III. (a) Distance from the bilateral femoral artery to the xiphoid process minus different range;(b) Distance from the bilateral femoralartery to the umbilicus plus different range;(c) Distance from the bilateral femoralartery to the umbilicus plus the 4 equal distances between xiphoid and umbilicus. 